\title{
NSM SOLUTION ON TRANSIENT NATURAL CONVECTIVE VISCOUS FLUID FLOW PAST A VERTICAL CONE
}

\author{
Y. Immanuel ${ }^{1}$, Bapuji Pullepu ${ }^{2}$, M. Selva Rani ${ }^{2}$ \\ ${ }^{1}$ Department of Mathematics \\ Sathyabama University \\ Chennai, INDIA \\ ${ }^{2}$ Department of Mathematics \\ SRM University \\ Kattankulathur, INDIA
}

\begin{abstract}
The unsteady free convective flow of the non-isothermal vertical cone with wall surface temperature varying as power function of distance from the apex $(x=0)$ with the effects of viscous dissipation is considered here. The dimensionless parabolic integro-partial differential equations that are non-linear, unsteady and coupled are solved using a new method NSM technique. The velocity, temperature profiles and the local as well as average skinfriction and Nusselt number have been studied and analyzed graphically for the effect of viscous dissipation for various parameters of angle $\phi$, Prandtl number $\operatorname{Pr}$ and $n$ (exponent in power law variation in surface temperature). The present results are compared with available results in literature and are found to be in good agreement.
\end{abstract}

AMS Subject Classification: 80M20, 76D10, 76M20, 74S30

Key Words: natural convection, network simulation method, non-uniform surface temperature, PSPICE, vertical cone, viscous dissipation

\section{Introduction}

Natural convection flows under the influence of gravitational force have been

Received: February 13, 2016

Published: June 20, 2016

${ }^{\S}$ Correspondence author (c) 2016 Academic Publications, Ltd.

url: www.acadpubl.eu 
investigated most extensively because they occur frequently in nature as well as in science and engineering applications. When a heated surface is in contact with the fluid, the result of temperature difference causes buoyancy force, which induces the natural convection.

The irreversible process by means of which the work is done by a fluid on adjacent layers due to the action of shear forces transformed into heat is defined as viscous dissipation. The effect a gas in space and in geological processes, and in fluids internal to various bodies. Viscous mechanical dissipation effects are usually characterized by the Eckert number. Viscous dissipation effects can be measured by using an independent parameter called dissipation number. of viscous dissipation in natural convection is appreciable when the induced kinetic energy becomes appreciable when compared to the amount of heat transferred. This occurs when either the equivalent body force is large or when the convection region is extensive. Viscous dissipation occurs in natural convection in natural devices. Such dissipation effects may also be observed in the presence of strong gravitational fields and in processes wherein the scale of the process is very large, e.g., on large planets, in large masses of Taking this into account, viscous dissipative heat is included in the energy equation. The heat due to viscous dissipation in the energy equation is very small and is usually neglected. However, if the gravitational force is intensive or if the Prandtl number of the fluid is very high, the viscous dissipative effects cannot be neglected.

Laminar free convection boundary layer flow and heat transfer over a vertical cone have been the subject of interest of many investigators because they occur frequently in nature as well as in industrial and technological applications. It is observed that significant viscous dissipation may occur in free convection in various equipments due to large amount of decelerations or which function at high rotational speed. Heat transfer problem arises mainly with the design of spacecrafts i.e. aerodynamic heating in the thin boundary layer around high speed aircraft raises the temperature of the skin.

Since 1953, several authors $[1,2,3,4,5,6,7,8,9,10,11,12,13,14,15,16$, 17], have studied the free convective flow past a cone / truncated cone / rotating cone in porous / Non porous medium with different boundary conditions by applying Magnetic field, Radiation, Chemical reaction, Heat generation / absorption etc.,

The numerical tool employed to solve this problem is the Network Simulation Method (NSM). NSM is a hybrid differential-difference method that uses a real continuous time variable in such a way that no time interval needs to be established by the users to obtain the numerical solution. Hence, the immediate advantage of this method is that errors are quantified in terms of the number 
of control volumes. NSM originated from the theory of electric circuits, more specifically electro-thermal analogy ( $\mathrm{RC}$ analogy). Electrical and thermal systems are said to be analogous if they are formulated in the same domain with similar equations and identical initial and boundary conditions. The model is elaborated in network through space, not temporal, discretization. The NSM is a numerical technique for solving nonlinear heat conduction equations. Nevertheless, its capacity to implement in the model any kind of nonlinearity (due to boundary conditions, phase-change processes, temperature dependencies of the thermal properties, etc.) distinguishes NSM from other analogies generally presented in text books.

The network simulation method is a numerical technique whose accuracy, efficiency and reliability have already been proven in numerous linear and nonlinear direct problems in heat transfer engineering $[20,21,22,23,24,25,26,27$, $28,29,30,31]$. NSM simulates the behaviour of unsteady electric circuits by means of resistors, capacitors and non-linear devices that seek to resemble thermal systems governed by unsteady linear or non-linear equations. The whole network model, including the devices associated with the boundary conditions, is solved by the numerical computer code Pspice [18], and the graph solution can be obtained by means the Probe software of Pspice.

The NSM technique begins with the design of the network model of the element cell, following which we incorporate the boundary conditions. It should be mentioned that heat conservation is satisfied since Kirchhoff conservation law for the electric currents is inherent in the networks. In this way, no additional conditions are needed to ensure this condition. The uniqueness of the temperature variable is also satisfied due to Kirchhoff voltage law.

NSM yields the ordinary differential equations the basis ones for implementing standard electrical network model for an elemental control volume from the partial differential equations that define the mathematical model of physical process and by means of spatial discretization [19]. [20], represents the relation between NSM and heat transfer.

Bi-dimensional (axial-radial) wall and fluid heat conduction is considered and the effects of Peclet number and the pipe thickness are analyzed and a numerical tool NSM is applied to study the heat transfer transient-state related to a fully development laminar flow in circular pipes in [21]. [22], studied numerical solution of the heat conduction equation with the electro thermal analogy and the code PSPICE. [23], presented transient free convection MHD flow of a dissipative fluid along a semi-infinite vertical plate with mass transfer, the surface of which is exposed to a constant heat flux; The non-linear system of partial differential equations is numerically solved by means of the net- 
work simulation method, based on the thermo-electric analogy. The unsteady, buoyancy-induced, hydro magnetic, thermal convection flow in a semi-infinite porous regime adjacent to an infinite hot vertical plate moving with constant velocity is studied in the presence of significant thermal radiation and the solution is obtained using the NSM method in [24]. The steady, laminar axi-symmetric convective heat and mass transfer in boundary layer flow over a vertical thin cylindrical configuration in the presence of significant surface heat and mass flux is studied theoretically and numerically and the resulting equations are solved using the Network Simulation Method (NSM) for the buoyancy-assisted pure free convection and also the pure forced convection cases in [25]. The transient problem for fully developed mixed convection considering the effect of viscous dissipation is investigated for the laminar flow in a parallel-plate vertical channel by means of the Network Simulation Method and the thermal boundary condition considered is a forced convection in [26]. The laminar heat and mass transfer of an electrically-conducting, heat generating/absorbing fluid past a perforated horizontal surface in the presence of viscous and Joule (Ohmic) heating is solved using the NSM in [27]. [28], designed a suitable network model to model the equations of the unsteady problem (MHD free convection flow past a semi-infinite vertical porous plate), with the fluid considered non-gray (absorption coefficient dependent on wave length), and studied the effects of thermal radiation, viscous dissipation, suction/injection and magnetic field.

In [29], the steady-state, magneto hydrodynamic, optically thick, dissipative gas boundary layer flow and heat transfer past a non-isothermal porous wedge embedded in a scattering, homogenous, isotropic Darcy Forchheimer porous medium, with significant thermal radiation effects in the presence of heat sink/sources and surface transpiration, in an $(x ; y)$ coordinate system is solved using NSM. The effects of dependence on temperature of the viscosity and electric conductivity, Reynolds number and particle concentration on the unsteady MHD flow and heat transfer of a dusty, electrically conducting fluid between parallel plates in the presence of an external uniform magnetic field have

\section{Mathematical Analysis}

An axi-symmetric unsteady, laminar free convection flow of a viscous incompressible fluid past a vertical cone with non uniform surface temperature $T_{w}^{\prime}(x)=$ $T_{\infty}^{\prime}+a x^{n}$ and viscous dissipation is considered. It is assumed that the effects of pressure gradient along the boundary layer are negligible. It is also assumed 
that the cone surface and the surrounding fluid which is at rest are at the same temperature. Then at time $t^{\prime}>0$, the temperature of the cone surface is suddenly raised to $T_{w}^{\prime}(x)=T_{\infty}^{\prime}+a x^{n}$ and it is maintained, $n$ is the exponent in power law variation in surface temperature. The co-ordinate system is chosen (as shown in Fig. 1 such that $x$ measures the distance been investigated using the network simulation method (NSM) and the electric circuit simulation program Pspice in [30]. A theoretical study of unsteady magneto hydrodynamic viscous Hartmann-Couette laminar flow and heat transfer in a Darcian porous medium intercalated between parallel plates, under a constant pressure gradient is presented and Viscous dissipation, Joule heating, Hall current and ion slip current effects are included in [31].

The objective of the present investigation is to study the effects of viscous dissipation in an unsteady free convection flow from a non-isothermal vertical cone and it has not received any attention in literature. Hence, the present work studies and deals with the laminar free convection flow over a non-isothermal vertical cone. The governing boundary layer equations are solved by NSM with $\operatorname{Pr}, \phi, n$ and $\epsilon$ as controlling parameters. In order to check the accuracy of our numerical results, the present results in steady state at $X=1.0$ obtained and considering modified Grashoff number $G r_{L}^{*}=$ $G r_{L} \cos \phi=\frac{g \beta \cos \phi\left(T_{w}^{\prime}-T_{\infty}^{\prime}\right) L^{3}}{\nu^{2}}$ (i.e. the numerical solutions obtained from the Equations (11)-(13) using boundary conditions (14) are independent of cone semi vertical angle $\phi$ ) are compared with the available results of Hering and Grosh [3], Hering [4], and Pop et al. [9], are found to be in excellent agreement. along surface of the cone from the apex $(x=0)$ and $y$ measures the distance normally outward. Here $\phi$ is the semi vertical angle of the cone and $r(x)$ is the local radius of the cone. The fluid properties are assumed to be constant except for density variations which induce buoyancy force term in the momentum equation and it plays main role in free convection. The governing boundary layer equations of continuity, momentum, and energy under Boussinesq approximation are as follows:

Equation of continuity:

$$
\frac{\partial}{\partial x}(u r)+\frac{\partial}{\partial y}(v r)=0
$$

Equation of momentum:

$$
\frac{\partial u}{\partial t^{\prime}}+u \frac{\partial u}{\partial x}+v \frac{\partial u}{\partial y}=g \beta \cos \phi\left(T^{\prime}-T_{\infty}^{\prime}\right)+\nu \frac{\partial^{2} u}{\partial y^{2}}
$$


Equation of energy:

$$
\frac{\partial T^{\prime}}{\partial t^{\prime}}+u \frac{\partial T^{\prime}}{\partial x}+v \frac{\partial T^{\prime}}{\partial y}=\alpha \frac{\partial^{2} T^{\prime}}{\partial y^{2}}+\frac{\mu}{\rho C_{p}}\left(\frac{\partial u}{\partial y}\right)^{2}
$$

The initial and boundary conditions are

$$
\begin{array}{rll}
t^{\prime} \leq 0: u=0, & v=0, T^{\prime}=T_{\infty}^{\prime} & \text { for all } x \text { and } y \\
t^{\prime}>0: u=0, & v=0, T_{w}^{\prime}(x)=T_{\infty}^{\prime}+a x^{n} & \text { at } y=0 \\
u=0, & T^{\prime}=T_{\infty}^{\prime} & \text { at } x=0 \\
u \rightarrow 0, & T^{\prime} \rightarrow T_{\infty}^{\prime} & \text { as } y \rightarrow \infty
\end{array}
$$

Where $a$ is constant, $C_{p}$ is specific heat at constant pressure, $g$ is acceleration due to gravity, $n$ is exponent in the power law variation, $r$ is local cone radius, $T^{\prime}$ is temperature, $t^{\prime}$ is time, $u, v$ is velocity components in the $x$ and $y$ directions, $x, y$ are spatial coordinates, $\alpha$ is thermal diffusivity, $\beta$ is volumetric thermal expansion, $\phi$ is semi vertical cone angle, $\rho$ is density, $\mu$ is dynamic viscosity, $\nu$ is kinematic viscosity and the indices $w$ refers to the conditions on the wall and $\infty$ refers to free stream conditions.

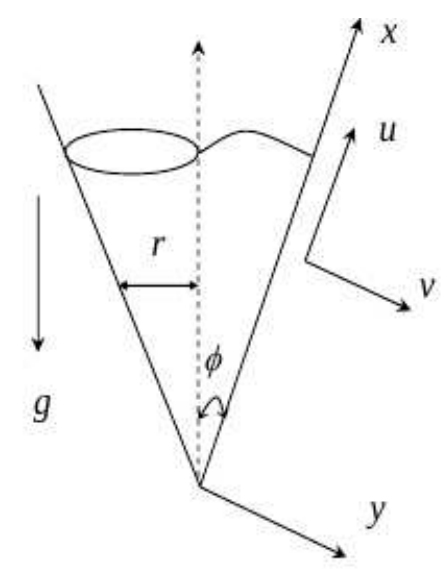

Fig. 1. Physical model and co-ordinate system

Local skin-friction and local Nusselt number are given respectively by

$$
\tau_{x}=\mu\left(\frac{\partial u}{\partial y}\right)_{y=0}
$$




$$
N u_{x}=\frac{-x\left(\frac{\partial T^{\prime}}{\partial y}\right)_{y=0}}{T_{w}^{\prime}-T_{\infty}^{\prime}}
$$

Average skin friction is given by

$$
\bar{\tau}_{L}=\frac{2 \mu}{L^{2}} \int_{0}^{L} x\left(\frac{\partial u}{\partial y}\right)_{y=0} d x
$$

Average heat transfer co-efficient over cone surface is

$$
\bar{h}=\frac{-2 k}{L^{2}} \int_{0}^{L} x \frac{\left(\frac{\partial T^{\prime}}{\partial y}\right)_{y=0}}{T_{w}^{\prime}-T_{\infty}^{\prime}} d x
$$

The average Nusselt number is

$$
\overline{N u} L=\frac{\bar{h} L}{k}=\frac{-2}{L} \int_{0}^{L} x \frac{\left(\frac{\partial T^{\prime}}{\partial y}\right)_{y=0}}{T_{w}^{\prime}-T_{\infty}^{\prime}} d x
$$

Where $h$ is average heat transfer coefficient over the cone surface, $k$ is thermal conductivity, $L$ is reference length, $N u_{x}$ is local Nusselt number, $N u_{L}$ is average Nusselt number, $\tau_{x}$ is local skin friction, $\tau_{L}$ is average skin friction.

Using the following non-dimensional quantities:

$$
\begin{gathered}
X=\frac{x}{L}, Y=\frac{y}{L}\left(G r_{L}\right)^{\frac{1}{4}}, R=\frac{r}{L}, \text { where } r=x \sin \phi \\
V=\frac{v L}{\nu}\left(G r_{L}\right)^{\frac{-1}{4}}, U=\frac{u L}{\nu}\left(G r_{L}\right)^{\frac{-1}{2}}, t=\frac{\nu t^{\prime}}{L^{2}}\left(G r_{L}\right)^{\frac{1}{2}} \\
T=\frac{T^{\prime}-T_{\infty}^{\prime}}{T_{w}^{\prime}-T_{\infty}^{\prime}}, G r_{L}=\frac{g \beta\left(T_{w}^{\prime}-T_{\infty}^{\prime}\right) L^{3}}{\nu^{2}}, \operatorname{Pr}=\frac{\nu}{\alpha}
\end{gathered}
$$

and $\epsilon=\frac{g \beta L}{C_{p}}$ where $\epsilon$ is viscous dissipation parameter as described in [23, 32].

Equations (1)-(3) are reduced to the following non dimensional form:

$$
\begin{gathered}
\frac{\partial(U R)}{\partial X}+\frac{\partial(V R)}{\partial Y}=0 \quad \text { (or) } \frac{\partial U}{\partial X}+\frac{\partial V}{\partial Y}+\frac{U}{X}=0 \\
\frac{\partial U}{\partial t}+U \frac{\partial U}{\partial X}+V \frac{\partial U}{\partial Y}=T \cos \phi+\frac{\partial^{2} U}{\partial Y^{2}} \\
\frac{\partial T}{\partial t}+U \frac{\partial T}{\partial X}+V \frac{\partial T}{\partial Y}=\frac{1}{P r} \frac{\partial^{2} U}{\partial Y^{2}}+\epsilon\left(\frac{\partial U}{\partial Y}\right)^{2}
\end{gathered}
$$


The corresponding non-dimensional initial and boundary conditions are

$$
\begin{array}{rrrl}
t \leq 0: U=0, & V=0, T=0 & \text { for all } X, Y \\
t>0: U=0, & V=0, T=X^{n} & \text { at } Y=0, \\
U=0, & T=0 & \text { at } X=0, \\
U \rightarrow 0, & T \rightarrow 0 & \text { as } Y \rightarrow \infty .
\end{array}
$$

Local skin-friction and local Nusselt number in non-dimensional quantities are

$$
\begin{gathered}
\tau_{X}=G r_{L}^{\frac{3}{4}}\left(\frac{\partial U}{\partial Y}\right)_{Y=0} \\
N u_{X}=\frac{X}{T_{Y=0}}\left(\frac{-\partial T}{\partial Y}\right)_{Y=0} G r_{L}^{\frac{1}{4}}
\end{gathered}
$$

Average skin friction and average Nusselt number in non-dimensional quantities are

$$
\begin{gathered}
\bar{\tau}=2 G r_{L}^{\frac{3}{4}} \int_{0}^{1} X\left(\frac{\partial U}{\partial Y}\right)_{Y=0} d X, \\
\overline{N u}=2 G r_{L}^{\frac{1}{4}} \int_{0}^{1} \frac{X}{T_{Y=0}}\left(\frac{-\partial T}{\partial Y}\right)_{Y=0} d X .
\end{gathered}
$$

Where $G r_{L}$ is Grashof number, $\operatorname{Pr}$ is Prandtl number, $R$ is non dimensional local radius, $T$ is non dimensional temperature, $t$ is non dimensional time, $U, V$ is non dimensional velocities in the $X$ and $Y$ directions, $X, Y$ is non dimensional spatial coordinates along the cone generator and along the normal to it.

\section{Solution Procedure}

The governing partial differential equations (11)-(13) which are unsteady, coupled and non-linear with initial and boundary conditions (14) are solved using a new method called the network simulation method.

The network simulation method is a numerical technique for solving nonlinear heat conduction equations whose accuracy, efficiency and reliability have already been proven in numerous linear and nonlinear direct problems in heat transfer engineering $[20,21,22,23,24,25,26,27,28,29,30,31]$. NSM is based on the classical electro-thermal analogy between thermal and electrical variables. Electrical and thermal systems are said to be analogous if they are formulated in the same domain with similar equations and identical initial and boundary conditions. The model is elaborated in network through space, not 
temporal, discretization. In the NSM technique, discretization of the differential equations is founded on the finite difference formulation, where discretization of the spatial coordinates is necessary, while time remains a real continuous variable. Hence, the imminent advantage of this method of non mathematical manipulations in the discretization of the time coordinate, is necessary for most of the numerical methods used currently, since the code does this work. This is an essential difference between the most classic methods and the NSM.

Two circuits (Fig. 1a and 1b) are developed for each non-dimensional boundary-layer equation. The fundamental flow variables, $U$ and $T$ are equivalent to the variable voltage, and their derivates are equivalent to the electric current. A sufficient number of networks are connected in series to form the whole medium and boundary conditions and added by means of special electrical devices. The entire network model, including the devices associated with the boundary conditions is designed. A few programming rules are needed since not many devices form the network, solved by the numerical computer code Pspice [18] and the graph solution can be obtained by means the Probe software of Pspice. This code is imposed and adjusted continuously automatically for the time-step to reach a convergent solution in each iteration, according to the given stability and convergence requirement.

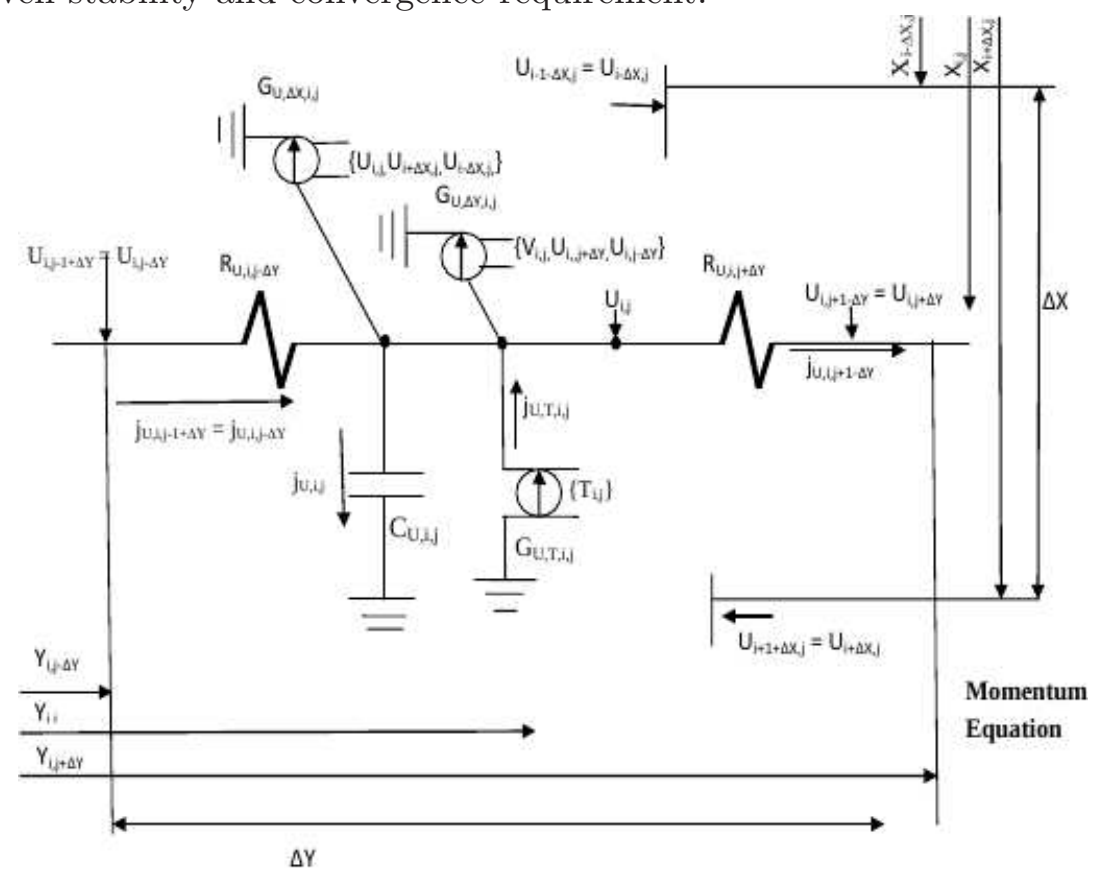

Fig.1a.Network model of the control volume - Momentum equation 


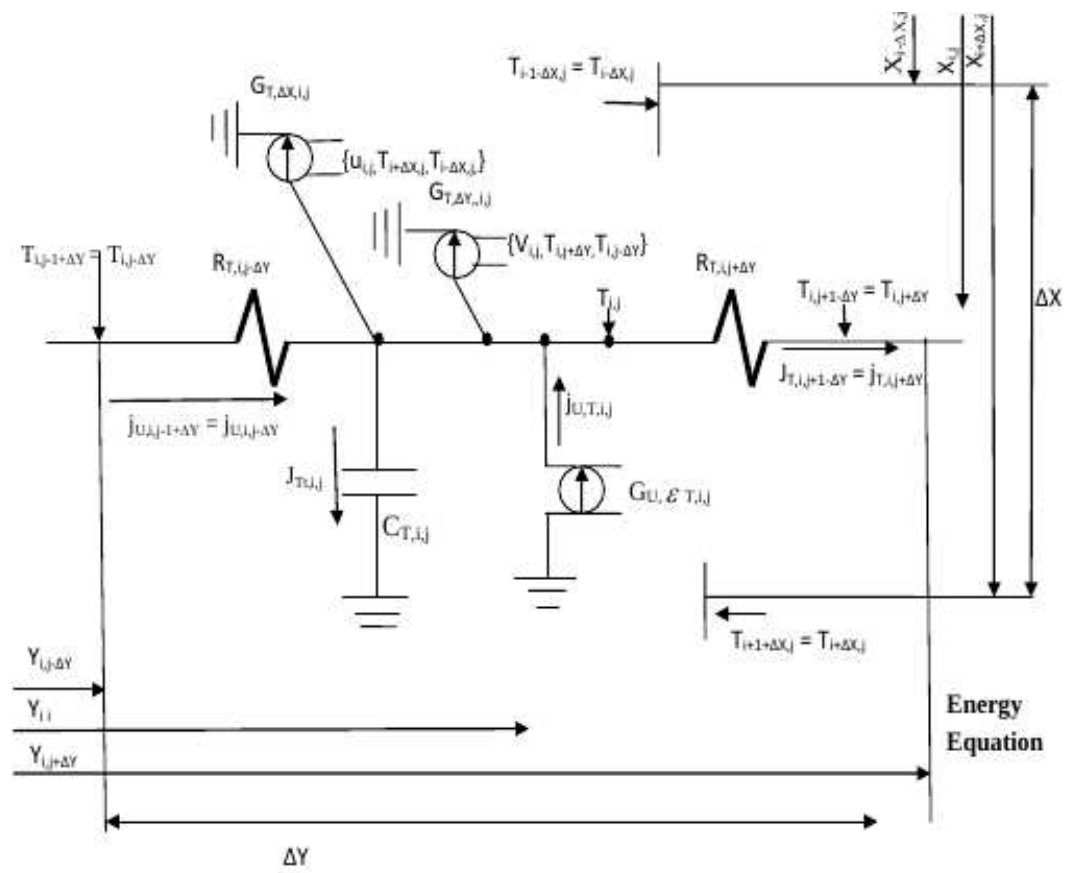

Fig. 1b.Network model of the control volume - Energy equation

To solve the set of non-linear differential equation (11)-(13) subject to boundary condition (14) the NSM has been applied. The finite-difference differential equations resulting from dimensionless continuity, momentum balance, energy balance equations are

$$
\begin{gathered}
\left(U_{i+\Delta X, j}-U_{i-\Delta X, j}\right) / \Delta X+\left(V_{i, j}-V_{i, j-\Delta Y}\right) /(\Delta Y / 2) \\
+U_{i, j} /(i \Delta X / 2)=0
\end{gathered}
$$

$$
\begin{aligned}
& \Delta Y d U_{i, j} / d t+\Delta Y U_{i, j}\left(U_{i+\Delta X, j}-U_{i-\Delta X, j}\right) / \Delta X \\
& +V_{i, j}\left(U_{i, j+\Delta Y}-U_{i, j-\Delta Y}\right)=\left(U_{i, j-\Delta Y}-U_{i, j}\right) /(\Delta Y / 2) \\
& \quad-\left(U_{i, j}-U_{i, j+\Delta Y}\right) /(\Delta Y / 2)+\Delta Y T_{i, j}
\end{aligned}
$$

$$
\begin{aligned}
& \Delta Y \operatorname{Prd} T_{i, j} / d t+\Delta Y \operatorname{Pr} U_{i, j}\left(T_{i+\Delta X, j}-T_{i-\Delta X, j}\right) / \Delta X \\
& +\operatorname{Pr} V_{i, j}\left(T_{i, j+\Delta Y}-T_{i, j-\Delta Y}\right)=\left(T_{i, j-\Delta Y}-T_{i, j}\right) /(\Delta Y / 2) \\
& \quad-\left(T_{i, j}-T_{i, j+\Delta Y}\right) /(\Delta Y / 2)+\operatorname{Pr} \epsilon\left(\left(U_{i+\Delta X, j}-U_{i-\Delta X, j}\right)^{2} / \Delta Y\right)
\end{aligned}
$$

In Eqs. (20) and (21) all the terms can be treated as a current. Therefore implementing Kirchhoff's law for electrical currents from circuit theory, the 
network model is obtained. To introduce the boundary conditions, voltage sources are employed to simulate constant values of velocity and temperature.

The NSM technique begins with the design of the network model of the element cell, following which we incorporate the boundary conditions. The following currents are defined

\subsection{Momentum Balance}

$$
\begin{aligned}
j_{U, i, j-\Delta Y} & =\left(U_{i, j-\Delta Y}-U_{i, j}\right) /(\Delta Y / 2) \\
j_{U, i, j+\Delta Y} & =\left(U_{i, j}-U_{i, j+\Delta Y}\right) /(\Delta Y / 2) \\
j_{U T, i, j} & =\Delta Y T_{i, j} \\
j_{U x, i, j} & =\Delta Y U_{i, j}\left(U_{i+\Delta X, j}-U_{i-\Delta X, j}\right) / \Delta X \\
j_{U y, i, j} & =V_{i, j}\left(U_{i, j+\Delta Y}-U_{i, j-\Delta Y}\right) \\
j_{U t, i, j} & =\Delta Y d U_{i, j} / d t
\end{aligned}
$$

where $j_{U, i, j+\Delta Y}$ and $j_{U, i, j-\Delta Y}$ are the currents that leave and enter the cell for the friction term of and are implemented by means two resistances $R_{U, i, j \pm \Delta Y}$ of values " $\Delta Y / 2$ "; $j_{U T, i, j}$ is the current due to the buoyancy term and $j_{U x, i, j}$ and $j_{U y, i, j}$ are the currents due to the inertia terms of $U$ and $V$ respectively and are implemented by means of voltage control current generators $G_{U, T, i, j}$, $G_{U, \Delta x, i, j}, G_{U, \Delta y, i, j}$. while $j_{U t, i, j}$ is the transitory term and is implemented by means one capacitor of value $C_{U, i, j}=\Delta Y$, connected to the centre of each cell.

\subsection{Energy Equation}

$$
\begin{aligned}
j_{T, i, j+\Delta Y} & =\left(T_{i, j}-T_{i, j+\Delta Y}\right) /(\Delta Y / 2) \\
j_{T, i, j-\Delta Y} & =\left(T_{i, j-\Delta Y}-T_{i, j}\right) /(\Delta Y / 2) \\
j_{T, i, j} & =\operatorname{Pr\epsilon }\left(\left(U_{i+\Delta X, j}-U_{i-\Delta X, j}\right)^{2} / \Delta Y\right) \\
j_{T x, i, j} & =\Delta Y \operatorname{Pr} U_{i, j}\left(T_{i+\Delta X, j}-T_{i-\Delta X, j}\right) / \Delta X \\
j_{T y, i, j} & =\operatorname{Pr} V_{i, j}\left(T_{i, j+\Delta Y}-U_{i, j-\Delta Y}\right) \\
j_{T t, i, j} & =\Delta Y \operatorname{Pr} d T_{i, j} / d t
\end{aligned}
$$

where $j_{T, i, j+\Delta Y}$ and $j_{T, i, j-\Delta Y}$ are the currents that leave and enter the cell for the friction term of $U$ and are implemented by means two resistances $R_{T, i, j \pm \Delta Y}$ 
of values " $\Delta Y / 2$ "; $j_{T, i, j}$ is the current due to the buoyancy term and $j_{T x, i, j}$ and $j_{T y, i, j}$ are the currents due to the inertia terms of $U$ and $V$ respectively and are implemented by means of voltage control current generators . while is the transitory term and is implemented by means one capacitor of value , connected to the centre of each cell.

A more rigorous step-by-step numerical analysis is described by GonzalezFernandez and Alhama [20], Zueco [23].

The finite difference equation corresponding to equation (11) is

$$
\begin{gathered}
V_{i, j}=\left(U_{i-\Delta X, j}-U_{i+\Delta X, j}\right) \Delta Y /(2 \Delta X) \\
-U_{i, j} \Delta Y /(i \Delta X)+V_{i, j-\Delta Y}
\end{gathered}
$$

Above Equations [(20) - (21)] can be written in the form of Kirchhoff's law as

$$
\begin{gathered}
j_{U, i, j+\Delta Y}-j_{U, i, j-\Delta Y}-j_{U T, i, j}+j_{U x, i, j}+j_{U y, i, j}+j_{U t, i, j}=0 \\
j_{T, i, j+\Delta Y}-j_{T, i, j-\Delta Y}-j_{T, i, j}+j_{T x, i, j}+j_{T y, i, j}+j_{T t, i, j}=0
\end{gathered}
$$

It should be mentioned that heat conservation is satisfied since Kirchhoff conservation law for the electric currents is inherent in the networks. In this way, no additional conditions are needed to ensure this condition. The uniqueness of the temperature variable is also satisfied due to Kirchhoff voltage law.

Finally, to implement the velocity, temperature boundary conditions (at $X=0$ and as $Y \rightarrow \infty$ ) ground elements are employed. Constant current and constant voltage at $Y=0$ are utilized to simulate the non-uniform surface temperature $\left(T=X^{n}\right)$. As regards the initial condition, the voltages $U=T=0$ for $t \leq 0$ are applied to the two capacitors $C_{U, i, j}$ and $C_{T, i, j}$.

\section{Results and Discussion}

In order to prove the accuracy of our numerical results the present results in Steady state at $X=1.0, \epsilon=0, \operatorname{Pr}=0.7$, and considering $G r_{L}^{*}=G r_{L} \cos \phi=$ $\frac{g \beta \cos \phi\left(T_{w}^{\prime}-T_{\infty}^{\prime}\right) L^{3}}{\nu^{2}}$ are compared with available similarity solutions in the open literature. 


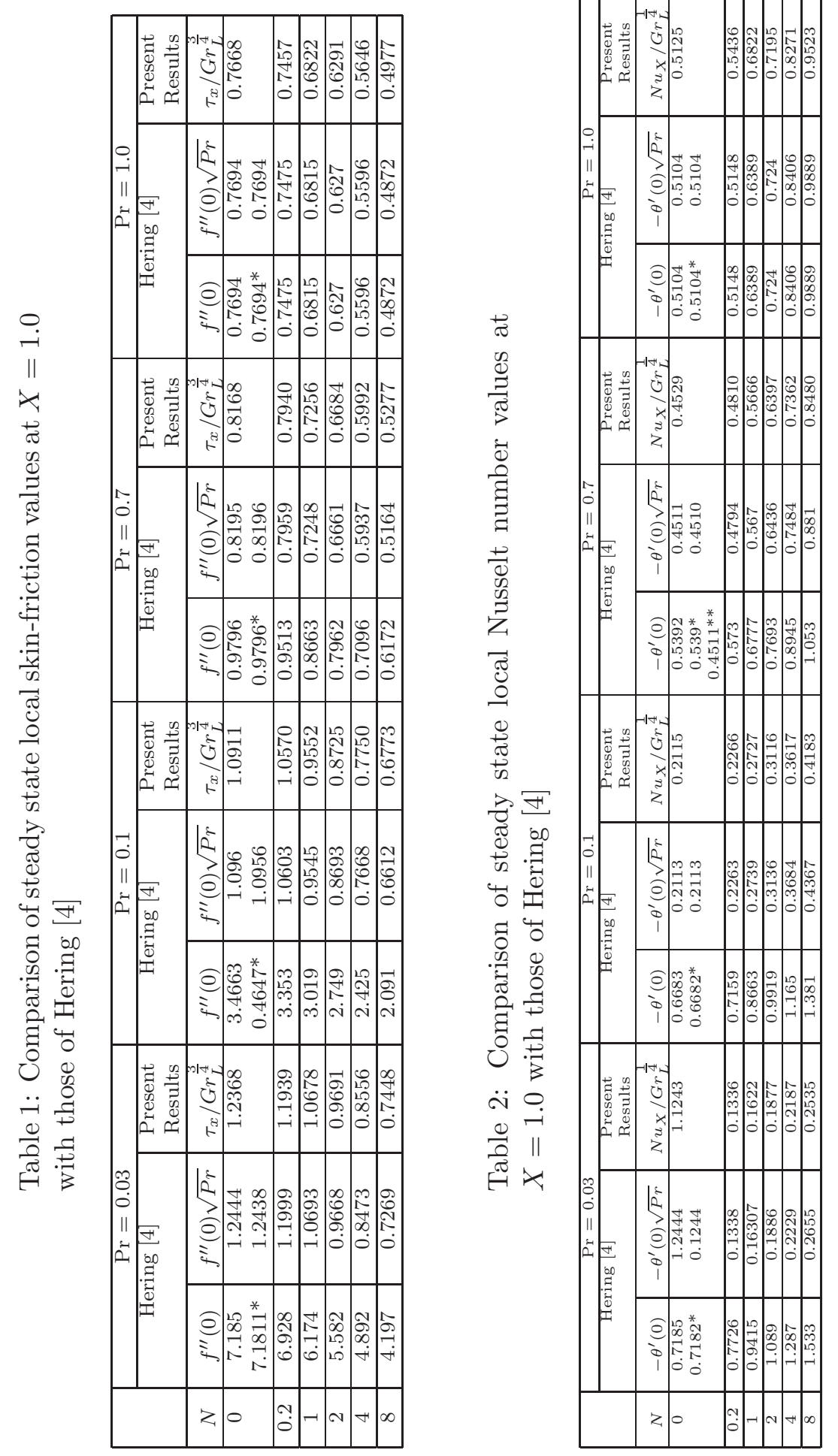


In addition, local skin-friction $\tau_{X}$ and local Nusselt number $N u_{X}$ values for different Prandtl number and exponent $n$ are compared with the results of Hering [4] in Table 1 and 2 respectively, where $f^{\prime \prime}(0),-\theta^{\prime}(0)$ are the local skin friction and the local Nusselt number obtained by Hering and Grosh [3] and Hering [4]. Here $*$ indicates the values obtained for pure free convection at $\xi=1$ by Pop et al. [9] in Table 1, 2 and ** indicates the values obtained at $X=1.0$ for free convection by Hering and Grosh [3] in Table 2. $f^{\prime \prime}(1,0),-\theta^{\prime}(1,0)$ are the local skin friction and the local Nusselt number obtained by Pop et al. [9] for free convection flow past a vertical cone at $\epsilon=1$. It is observed that the results are in good agreement with each other for small values of $n$. There is small deviation with these results for large values of $n$ and the deviation increases with $n$. Also present values of local skin-friction $\tau_{X}$ and local Nusselt number $N u_{X}$ values for different Prandtl number and exponent value $n$ are compared with the recent results. Finally, Pop and Thakar [7] results are for incompressible fluid and are same as those of Hering and Grosh [3]. Hence present results well agree with the results of Pop and Thakar [7] and for incompressible fluid.

In Figs. 2a and 2b transient velocity and temperature profiles are plotted at $X=1.0$ for various values of $\phi$ with $\operatorname{Pr}=0.71, \epsilon=0.1$ and $n=0.5$. From fig 2a, velocity decreases with the increasing values of $\phi$ and from fig $2 \mathrm{~b}$, Temperature increases with the increasing values of $\phi$.
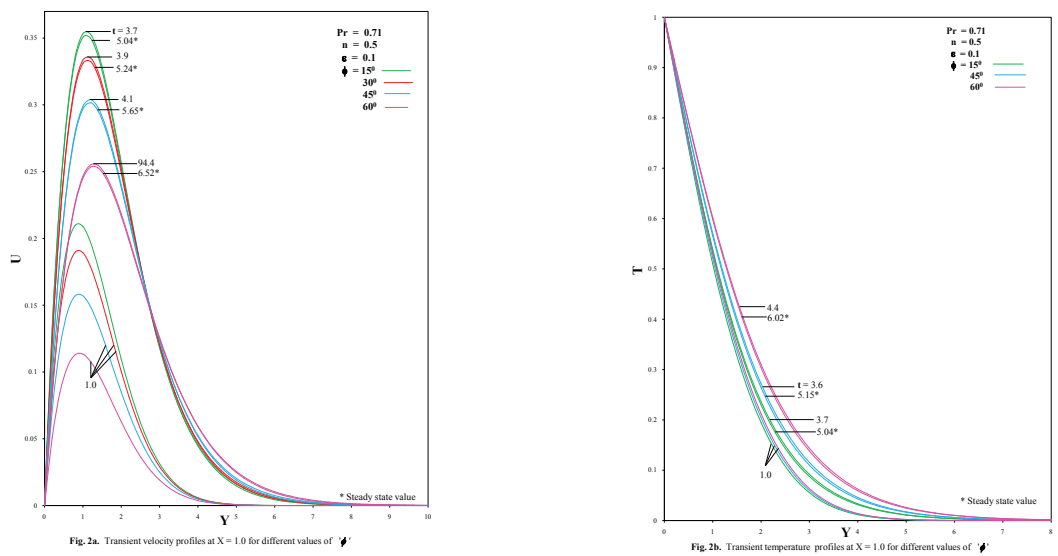

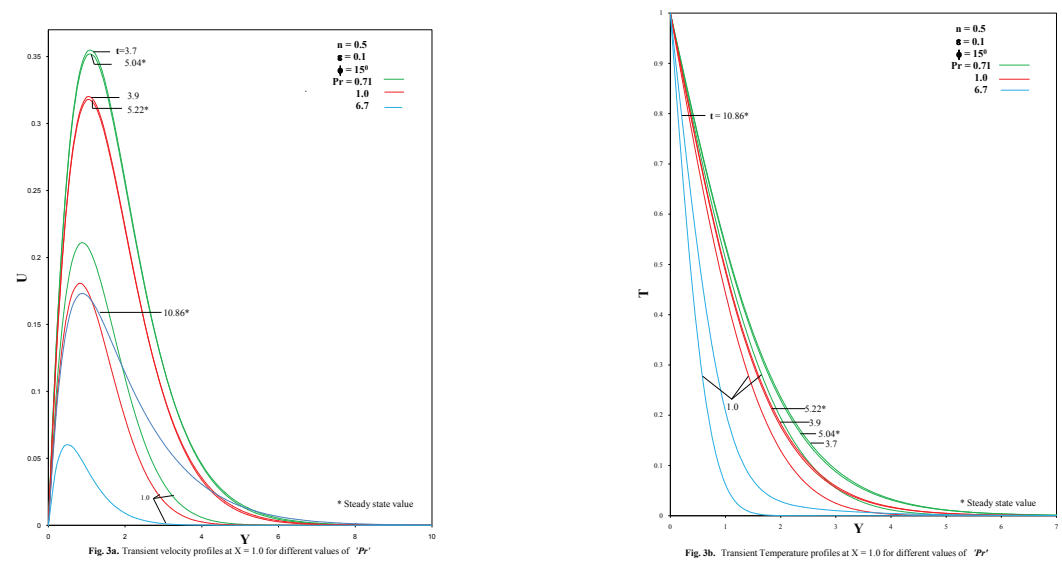

In Figs. 3a and $3 \mathrm{~b}$ transient velocity and temperature profiles are plotted at $X=1.0$ for various values of with $\epsilon=0.1$ and $n=0.5, \phi=15$. Viscous force increases and thermal diffusivity reduces with increasing $\operatorname{Pr}$ causes a reduction in the velocity and temperature. It is observed from the figures that the difference between temporal maximum values and steady state values are reduced when $P r$ increases. It is also noticed the time taken to reach steady state increases and thermal boundary layer thickness reduces with increasing $\mathrm{Pr}$. It is also clear from the Fig. 3a the momentum boundary layer thickness increases with the increase of $\mathrm{Pr}$.
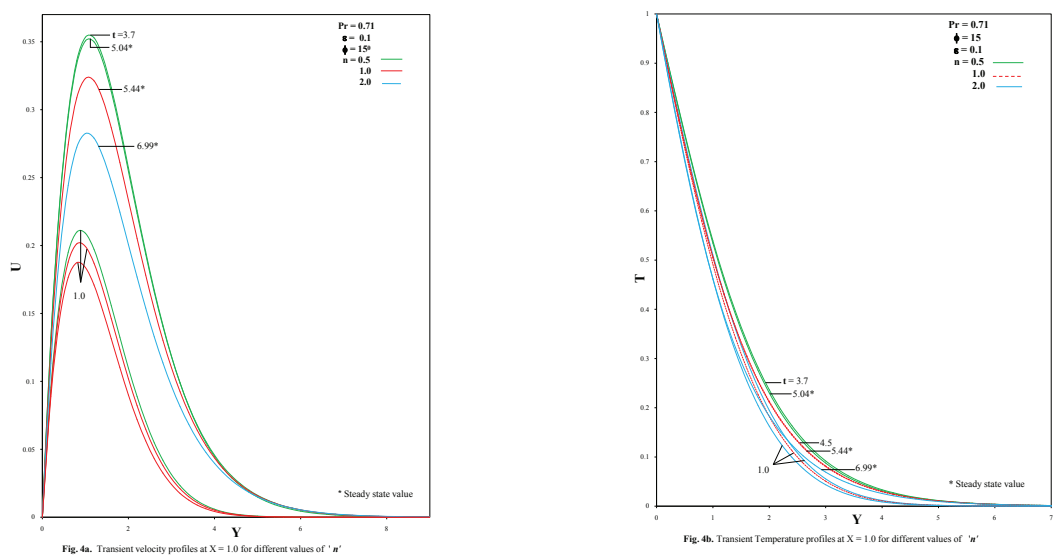

In Figs. 4a and 4b transient velocity and temperature profiles are shown at 
$X=1.0$ for various values of ' $n$ ' with $\operatorname{Pr}=0.71, \epsilon=0.1, \phi=15$. Impulsive forces are reduced along the surface of the cone near the vertex for increasing values of ' $n$ '. Due to this the difference between temporal maximum values and steady state values reduce. Velocity and temperature reduces and the time taken to reach steady state value increases, momentum and thermal boundary layer becomes thick for higher values of ' $n$ '.
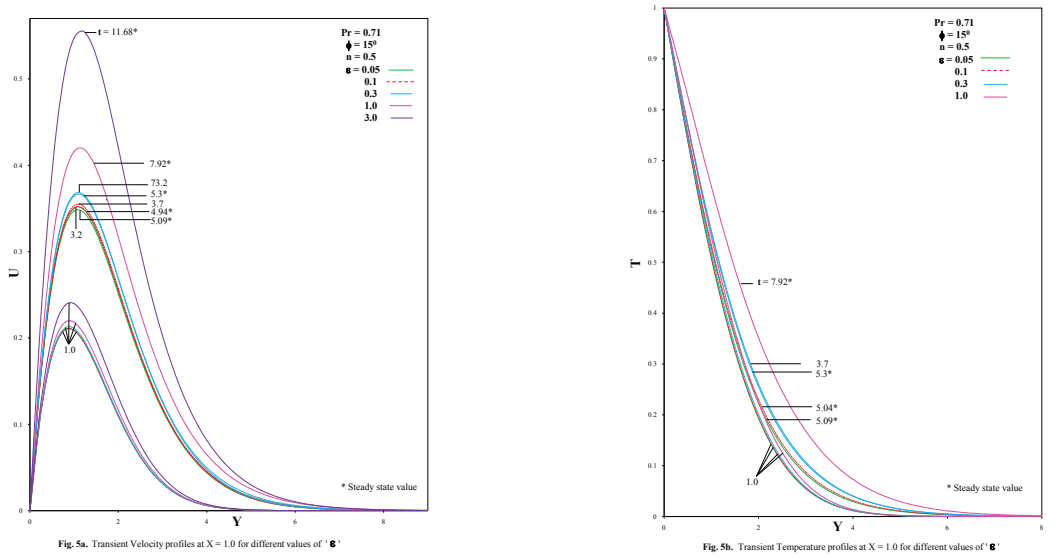

In Figs. 5a and 5b transient velocity and temperature profiles are shown at $X=1.0$ for various values of $\epsilon$ with $\operatorname{Pr}=0.71, n=0.5, \phi=15$. Greater viscous dissipative heat causes a rise in the temperature due to this as $\epsilon$ increases velocity and temperature increases. Also it is observed that the difference between temporal maximum values and steady state values are reduced while the time taken to reach steady state, thermal boundary layer and momentum boundary layer thickness increases with increasing $\epsilon$. 

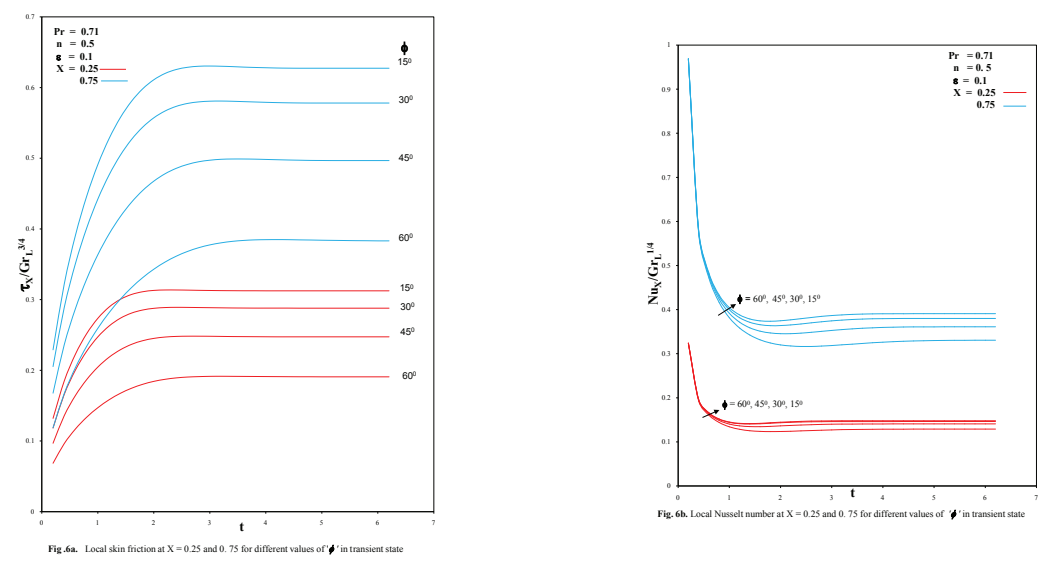

Figs $6 \mathrm{a}$ and $6 \mathrm{~b}$ depicted the local skin friction and Nusselt number for different values of $\Phi$ in the transient period at various positions on the surface of the cone $(X=0.25$ and 0.75$)$. It is observed that the local skin friction decreases for the increase in the values of $\Phi$ and local nusselt number also has the same effect.
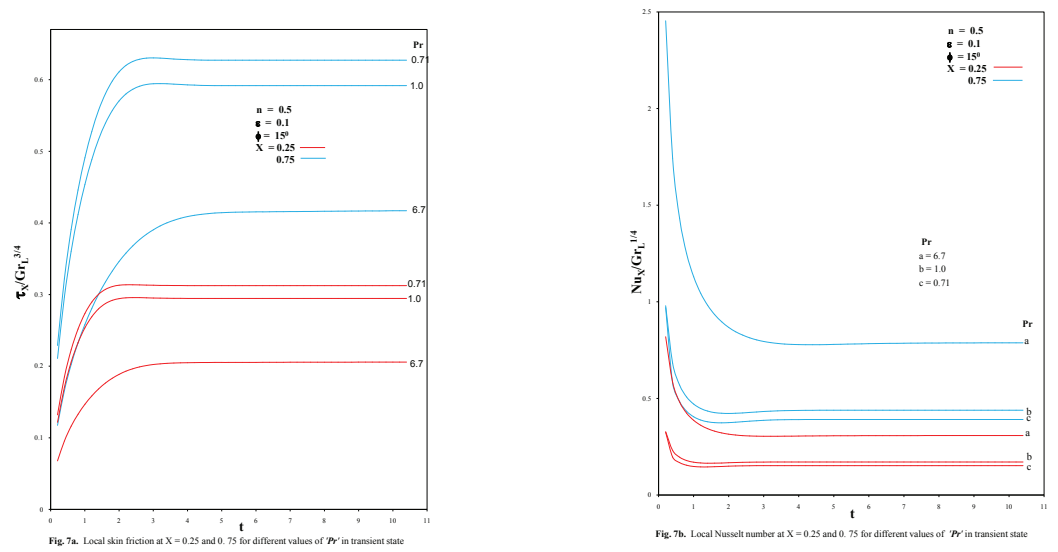

It is observed from the figures $7 \mathrm{a}$ and $7 \mathrm{~b}$ that local skin-friction $\tau_{X}$ and local Nusselt number $N u_{X}$ decreases with increasing $\operatorname{Pr}$ and also clear from the figures $8 \mathrm{a}$ and $8 \mathrm{~b}$ decreasing rate of $\tau_{X}$ and $N u_{X}$ increases when the distance increase from the cone vertex along the surface of the cone. 

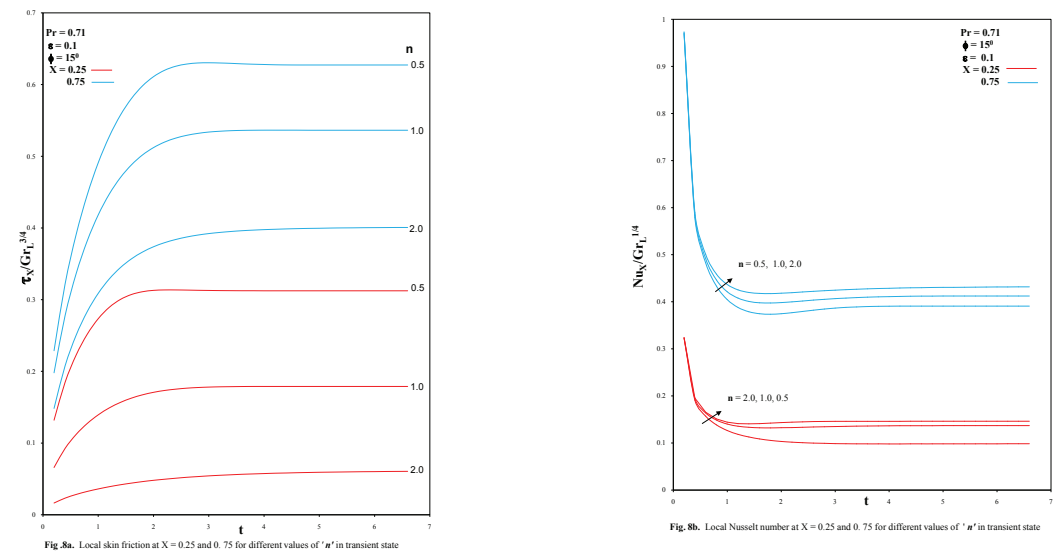

The variation of the local skin-friction $\tau_{X}$ and the local Nusselt number $N u_{X}$ in the transient period at various positions on the surface of the cone $(X=0.25$ and 0.75 ) and for different values of $\mathrm{n}$ are shown in Figs. 8a and $8 \mathrm{~b}$. It is observed that the local skin-friction decreases with increasing $n$ and the effect of $n$ over the local skin-friction is more near the apex of the cone and reduces gradually with increasing the distance along the surface of the cone from the apex. Also it is noticed that near the apex the local Nusselt number $N u_{X}$ reduces with increasing ' $n$ ', but that trend is slowly changed and reversed as distance increases along the surface from the apex.
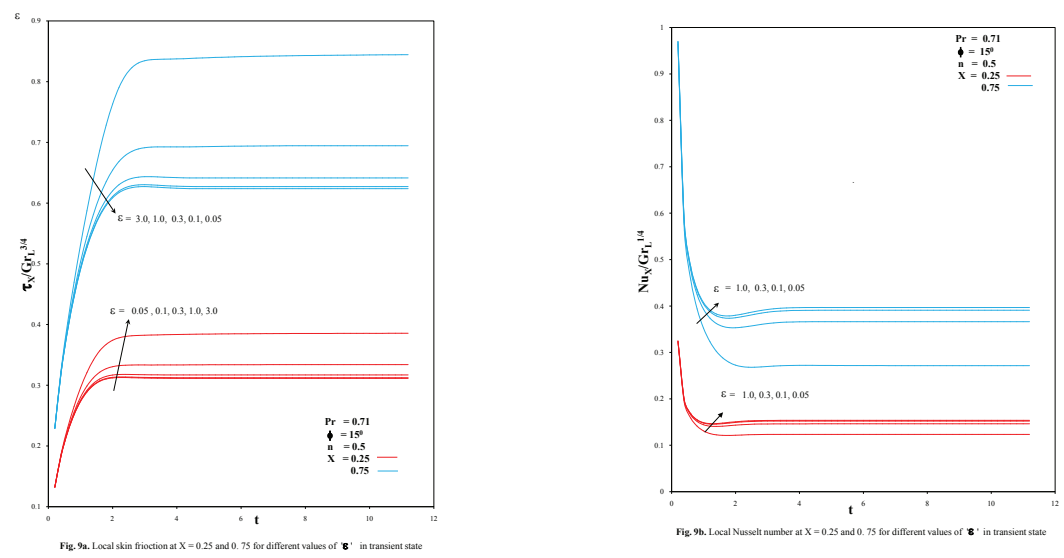
Fig. $9 \mathrm{a}$ and $9 \mathrm{~b}$ depicts the variation of local skin friction $\tau_{X}$ and local Nusselt umber $N u_{X}$ in the transient period at various positions on the surface of the cone $(X=0.25$ and 0.75$)$ for different values of $\epsilon$. The local wall shear stress decreases with decreasing values of $\epsilon$ but that trend is slowly changed and reversed as distance increases along the surface of the cone from the apex. The local rate of heat transfer decreases as $\epsilon$ increases. That is the rate of heat transfer is less near the cone apex and it increases along the surface of the cone from the apex.
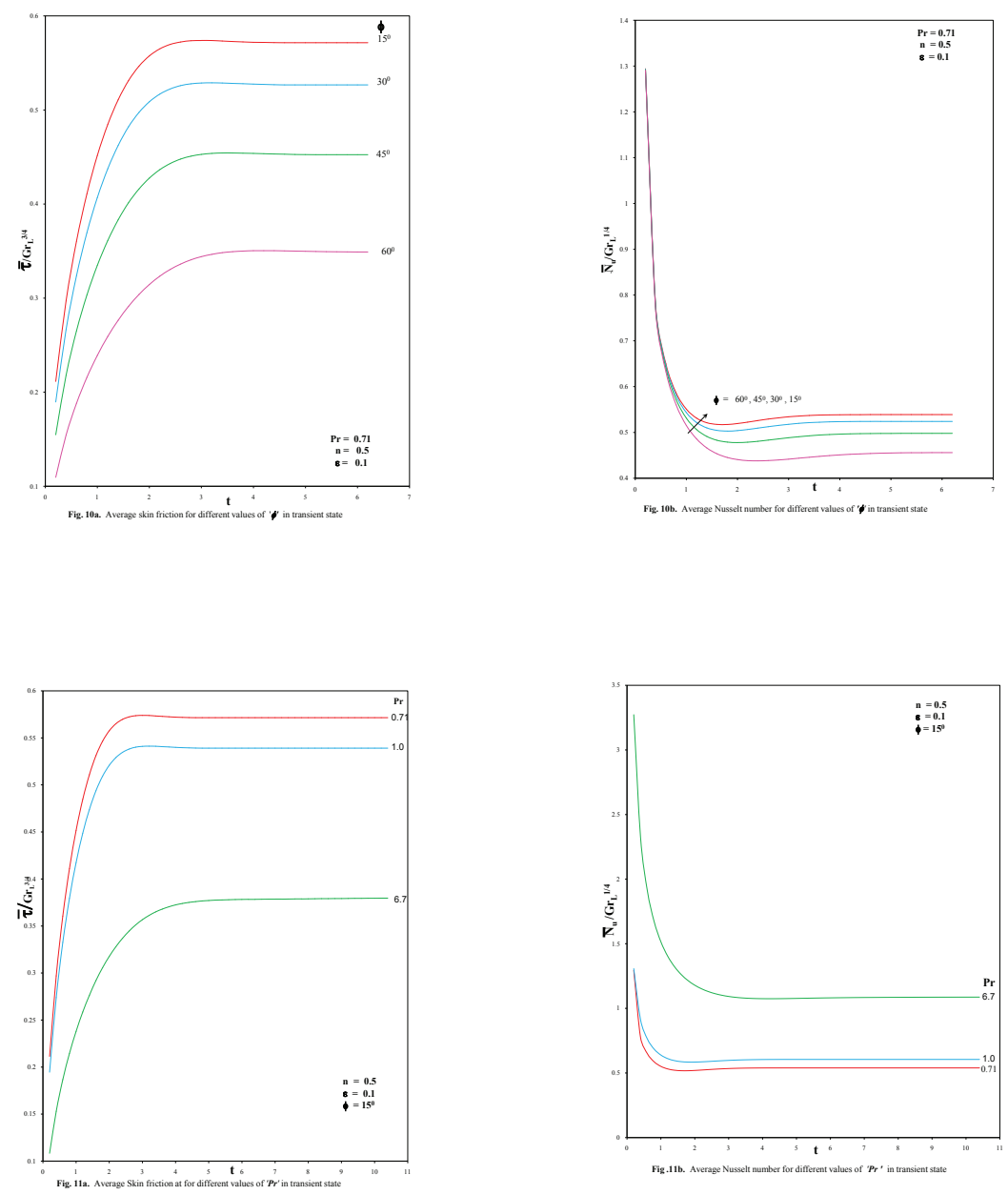

The variation of the average skin-friction $\bar{\tau}$ and average Nusselt number $\overline{N u}$ 
in transient period for various values of $\Phi, P r, n$ and $\epsilon$ are shown in Figs. 10a through $13 \mathrm{~b}$ respectively.

The influence of average skin-friction $\bar{\tau}$ is more in transient period for smaller values of $\Phi, \operatorname{Pr}$ and $n$ but the trend is reversed for the value of $\epsilon$. It is clear that $\overline{N u}$ is more for larger values of $\operatorname{Pr}, n$ and $\epsilon$ but the trend is reversed for the value of $\phi$.
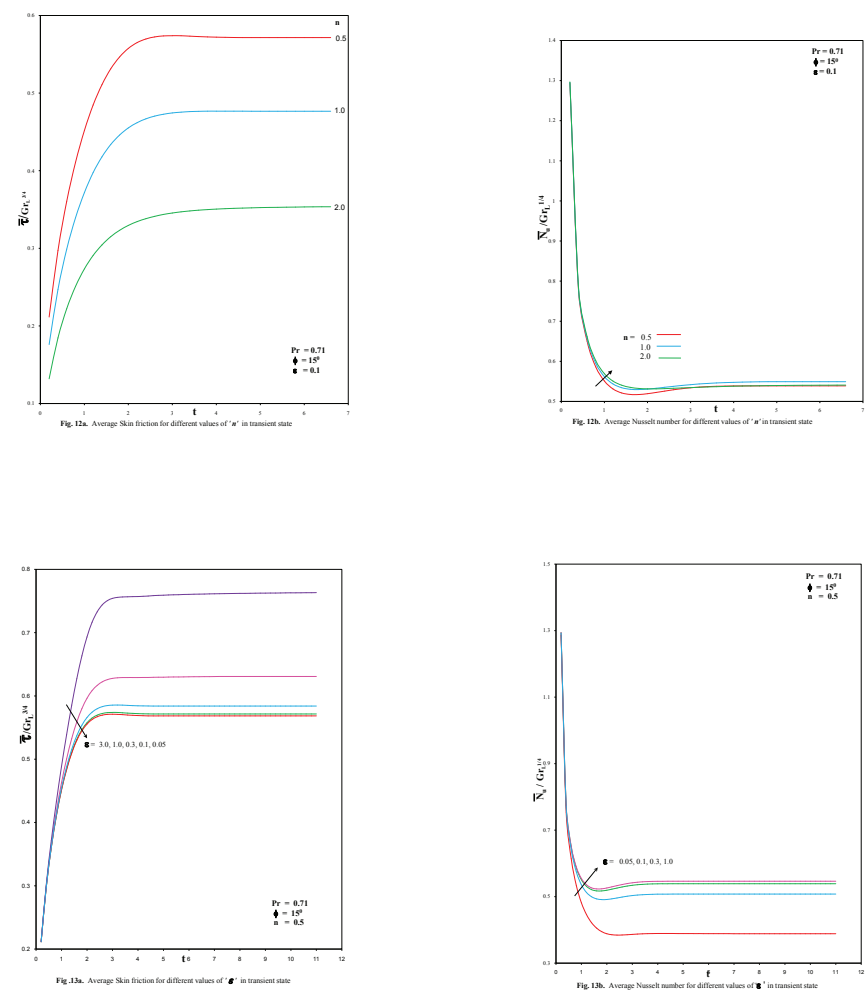

\section{Conclusions}

A numerical study has been carried out for the flow past an isothermal vertical cone. The dimensionless governing boundary layer equations are solved by an Network simulation method. The following conclusions are drawn:

- The velocity reduces for higher values of $\phi, \operatorname{Pr}, n$ and lower values of $\epsilon$.

- Temperature increases for higher values of $\phi, \epsilon$ and lower values of $\operatorname{Pr}$ 
and $n$.

- Momentum boundary layer becomes thick when $\operatorname{Pr}, n$ and $\epsilon$ are increased and the reverse effect for $\phi$.

- Thermal boundary layer becomes thin for lower values of $n, \epsilon$ and higher values of $\operatorname{Pr}$ and the reverse effect for $\phi$.

- The time taken to reach steady state increases with increasing $\phi, P r, n$ and $\epsilon$.

- The local skin-friction $\tau_{X}$ reduces for higher values of $\phi, \operatorname{Pr}, n$ and lower values of $\epsilon$.

- The local Nusselt number $N u_{X}$ decreases for larger values of $\phi, P r, n$ and $\epsilon$.

- The average skin friction $\bar{\tau}$ is more for smaller values of $\phi, \operatorname{Pr}, n$ and larger values of $\epsilon$.

- The average Nusselt number $N u$ is more for smaller values of $\phi$ and larger values of $\operatorname{Pr}, n$ and $\epsilon$.

\section{References}

[1] H.J. Merk and J.A. Prins, Thermal convection in laminar boundary layers I, Appl. Sci. Res., 4 (1953), 11-24.

[2] H.J. Merk and J.A. Prins, Thermal convection in laminar boundary layers II, Appl. Sci. Res., 4 (1954), 195-206.

[3] R.G. Hering and R.J. Grosh, Laminar free convection from a non-isothermal cone, Int. J. Heat Mass Transfer, 5 (1962), 1059-1068.

[4] R.G. Hering, Laminar free convection from a non-isothermal cone at low Prandtl number, Int. J. Heat Mass Transfer, 8 (1965), 1333-1337.

[5] K.T. Yang, J.L. Novotny and Y.S. Cheng, Laminar free convection from a non-isothermal plate immersed in a temperature stratified medium, Int. J. Heat Mass Transfer, 15 (1972), 1097-1109.

[6] H.S. Takhar and V.M. Soundalgekar, Combined convection past a vertical semi infinite plate with viscous dissipation, Mech. Res. Commun., 14(5/6) (1987), 307-315.

[7] I. Pop and H.S. Takhar, Compressibility effects in laminar free convection from a vertical cone, Appl. Sci. Res., 48 (1991), 71-82.

[8] M.A. Hossain and S.C. Paul, Free convection from a vertical permeable circular cone with non-uniform surface temperature, Acta Mech., 151 (2001), 103-114. 
[9] I. Pop, T. Grosan and M. Kumari, Mixed convection along a vertical cone for fluids of any Prandtl number: case of constant wall temperature, Int. J. of Numerical Methods for Heat and Fluid Flow., 13(7) (2003), 815-829.

[10] Bapuji Pullepu, K. Ekambavanan and A.J. Chamkha, Unsteady laminar natural convection flow past an isothermal vertical cone, Int. J. Heat and Technology, 25(2) (2007), $17-27$.

[11] Bapuji Pullepu, K. Ekambavanan and I. Pop, Finite difference analysis of aminar free convection flow past a non isothermal vertical cone, Heat Mass Transfer., 44(5) (2008), $517-526$.

[12] Bapuji Pullepu, K. Ekambavanan and A.J. Chamkha, Laminar natural convection from a non-isothermal vertical cone, Nonlinear Anal. Model. Control., 13(1) (2007), 47-60.

[13] S.G. Mohiddin, S. Vijayakumar Verma and N.Ch.S.N. Iyengar, Radiation and mass transfer effects on MHD free convective flow past a vertical cone with variable surface conditions in the presence of viscous dissipation, Int. Electr. Eng. Math. Soc., 8 (2010), $22-37$.

[14] P.M. Kishore, V. Rajesh, and S. Vijayakumar Verma, Viscoelastic buoyancy-driven MHDfree convective heat and mass transfer past avertical cone with thermal radiation and viscous dissipation effects, Int. J. Math. Mech., 6(15) (2010), 67-87.

[15] P.M. Kishore, V. Rajesh and S. Vijayakumar Verma, The effects of thermal radiation and viscous dissipation on MHD heat and mass diffussion flow past a surface embedded in a porous medium, Int. J. of Appl. Math and Mech., 6(11) (2010), 79-97.

[16] Bapuji pullepu, A.J. Chamkha and I. Pop, Unsteady laminar free convection flow past a non-isothermalvertical cone in the presence of a magnetic field, Chem. Eng. Commun., 199(3) (2012), 354-367.

[17] S.M.M. EL-Kabeir and E.A. EL-Sayed, Effects of thermal radiation and viscous dissipation on MHD viscoelastic free convection past a vertical isothermal cone surface with chemical reaction, Int. J. Energy. Tech., 4(10) (2012), 1-7.

[18] L.W. Nagel, PSPICE, A Computer Program to Simulate Semiconductor Circuits, Chapters 4, 5, 6, Memo UCB/ERL M520, PhD Thesis, University of California.

[19] K. Rektorys, The method of discretization in time for PDE, D. Reidel Publishers, Dordrechet, The Netherlands, 1982.

[20] F. Alhama and C.F. Gonzalez-Fernandez, Heat Transfer and the Network simulation method, NSM, Research signpost, Trivandrum (2002), 35-58.

[21] J. Zueco, F. Alhama and C.F. Gonzalez-Fernandez, Analysis of laminar forced convection with network simulation in thermal entrance region of ducts, Int. J. Thermal Science, 43(5) (2004), 443-451.

[22] F. Alhama, A. Campo and J Zueco, Numerical solution of the heat conduction equation with the electro-thermal analogy and the code PSPICE, Appl. Math. Compu., 162 (2005), $103-113$.

[23] J. Z. Jordan, Numerical study of an unsteady free convective MHD flow of a dissipative fluid along a vertical plate subject to a constant heat flux, Int. J. Engg. Sci., 44 (2006), $1380-1393$. 
[24] O. A. Beg, J. Zueco, S.K. Ghosh and HeidariAlireza, Unsteady MHD heat transfer in a semi-infinite porous medium with thermal radiation flux : Analytical Numerical Study, Adv. Numer. Anal. 2011(2011), 1-17.

[25] J. Zueco, O.A. Beg, H.S. Takhar and G. Nath, Network simulation of laminar convective heat and mass transfer over a vertical slender cylinder with uniform surface heat and mass flux, JAFM, 4(2)1 (2011), 13-23.

[26] J. Z. Jordan, Network method to study the transient heat transfer problem in a vertical channel with viscous dissipation, Int. commun. Heat Mass Transfer., 33 (2006), 10791087 .

[27] J. Zueco, O.A. Beg, H.S. Takhar and V.R. Prasad, Thermophoretic Hydromagnetic Dissipative Heat and Mass Transfer with Lateral Mass Flux, Heat Source, Ohmic Heating and Thermal Conductivity Effects Network Simulation Numerical Study, Appl. Therm. Eng., 29 (2009), 2808-2815.

[28] J. Z. Jordan, Network simulation method applied to radiation and viscous dissipation effects on MHD unsteady free convection over vertical porous plate, Appl. Math. Modelling., 31 (2007), 2019-2033.

[29] J. Zueco and O.A. Beg, Network simulation solutions for laminar radiating dissipative magneto-gas dynamic heat transfer over a wedge in non-Darcian porous regime, Math. Comput. Model., 50 (2009), 439-452.

[30] P. Eguia, J. Zueco, E. Granada and D. Patino, NSM solution for unsteady MHD Couette flow of a dusty conducting fluid with variable viscosity and electric conductivity, Appl. Math. Modelling., 35 (2011), 303-316.

[31] O.A. Beg, J. Zueco and H.S. Takhar, Unsteady magnetohydrodynamic HartmannCouette flow and heat transfer in a Darcian channel with Hall current, ionslip, viscous and Joule heating effects: Network numerical solutions, Commun. Nonlinear. Sci. Numer. Simulat., 14 (2009), 1082-1097.

[32] B. Gebhart, Effects of viscous dissipation in natural convection, J. Fluid Mech., 14 (1962), 225-232. 
